\title{
Kinematic Constraints for Assisted Single-Arm Manipulation
}

\author{
Tanya Tickel David Hannon Kevin M. Lynch Michael A. Peshkin J. Edward Colgate \\ Laboratory for Intelligent Mechanical Systems \\ Mechanical Engineering Department \\ Northwestern University \\ Evanston, IL 60208
}

\begin{abstract}
Of several possible forms of human-robot collaborative manipulation, we focus on the case where the human and the robot jointly manipulate a common load. In our formulation, the robot's role is to provide a constraint surface to guide the motion of the load. The value of this form of interaction, in terms of ergonomics, accuracy, or speed, depends on how humans make use of such constraints. We are studying natural single-arm manipulation of a load constrained to move along a guide rail. In this paper we present results of experiments showing that subjects apply significant forces against the rail, depending on the configuration of the arm and the orientation of the rail. These forces are unnecessary for the manipulation task, and we hypothesize that humans apply forces against the constraint to simplify the manipulation task.
\end{abstract}

\section{Introduction}

To design robots for human-robot collaborative manipulation, it is necessary to understand the human half of the system to ensure the safety of the operator and the overall effectiveness of the system. The design and control of human-interactive robots should take into consideration human motion preferences, ease and intuitiveness of the interaction, stress at joints, fatigue, etc. Our interest is in designing and controlling assist robots to make manipulation of heavy loads faster, more comfortable, and less likely to result in work-related musculoskeletal disorders (WMSD's).

We are investigating the use of passive robotic guides to assist a human in manipulating a load from one configuration to another. A guide acts as a workless, frictionless rail which confines the load to a one-dimensional curve in its configuration space. A robot implementing a passive guide, for example a cobot [15], does not amplify human muscle power, but simply redirects the momentum of the load without affecting the energy. The presence of the guide assists by minimizing tedious fine positioning required of the human. In addition, the guide limits the object to one motion freedom; all other freedoms are force freedoms. This allows the operator to choose the most comfortable force combination in this space while the guide directs the load to the goal. If certain muscles begin to fatigue, the operator may choose different force combinations, or the shape of the guide constraint can be modified. Since the robot guide is passive, it is as safe to interact with as a physical rail.

To design effective guides, we are studying how humans naturally manipulate a load confined to a frictionless guide rail. Such tasks are common in everyday life, including opening a door or a sliding drawer, turning a crank, or pedaling a bicycle. We hypothesize that interaction forces with constraints are stereotypical across subjects, and therefore amenable to analysis and modeling. A model of "natural" or "comfortable" interactions with a kinematic constraint may allow us to design guides that are easy to use and interact with, where ease may be defined by minimum metabolic cost, maximum endurance time, etc. ${ }^{1}$

We begin our study of natural human interaction with a guide rail with the case of manipulation with a single arm in a horizontal plane. With the wrist immobilized, the arm can be treated as a $2 \mathrm{R}$ manipulator (shoulder and elbow). We have chosen this task because it is a multi-joint task, requiring the coordination of different muscle groups, and because it has been heavily studied for the case of unconstrained motion (full motion freedom) and isometric force application (complete constraint, or full force freedom). There has been very little work on the intermediate case (one motion and one force freedom), which is the case of interest for assisted manipulation. An exception is a recent study by Svinin $e t$ al. [13] on natural interaction with a crank, using methods similar to those in this paper.

We have designed two sets of experiments. In both experiments, the subject holds a handle attached to a smooth linear rail. In the first set of experiments, the subject is asked to hold the handle stationary while the handle is pulled with different forces tangential to the rail. We are interested in the forces applied by the subject normal to the rail, which

${ }^{1} \mathrm{~A}$ related possibility is to design guides such that natural interaction will minimize the likelihood of WMSD's.

Kinematic constraints for assisted single-arm manipulation

T. Tickel, D. Hannon, K. M. Lynch, Michael A. Peshkin, and J. E. Colgate

IEEE International Conference on Robotics and Automation 2002 


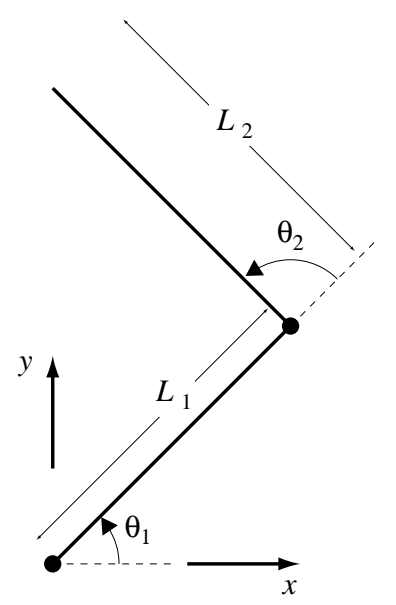

Figure 1: Kinematic model of the arm.

are not necessary for the task but may simplify the task for the subject. These experiments can help us build a model of natural static interaction with a constraint. In the second set of experiments, the subject is asked to quickly move the handle from one position to another, and we collect the trajectory and interaction force profiles. These data will allow us to build a dynamic model of interaction.

In this paper, we report the results of the first set of experiments, and compare the experimental results to theoretical predictions by different models of force generation.

\section{Planar Arm System}

All experiments were performed with the wrist held rigid and the shoulder at 90 degrees of abduction, i.e., in the horizontal plane of the shoulder. At these configurations, the arm is equivalent to a $2 \mathrm{R}$ planar manipulator, as shown in Figure 1. The joint torques $\tau=\left(\tau_{1}, \tau_{2}\right)^{T}$ are related to forces $\mathbf{f}=\left(f_{x}, f_{y}\right)^{T}$ at the hand by the relation

$$
\tau=J(\theta)^{T} \mathbf{f},
$$

where the Jacobian $J(\theta)$ is

$$
J(\theta)=\left(\begin{array}{rr}
-L_{1} \sin \left(\theta_{1}\right)-L_{2} \sin \left(\theta_{1}+\theta_{2}\right) & -L_{2} \sin \left(\theta_{1}+\theta_{2}\right) \\
L_{1} \cos \left(\theta_{1}\right)+L_{2} \cos \left(\theta_{1}+\theta_{2}\right) & L_{2} \cos \left(\theta_{1}+\theta_{2}\right)
\end{array}\right) .
$$

In the arm, joint torques are caused by a complex set of uniarticular muscles (crossing a single joint) and biarticular muscles crossing both the shoulder and the elbow $[1,16$, $8,5,11,9]$. Each muscle is capable of exerting contractile forces. The torque generated by each muscle is a function of the muscle tension due to muscle activation and the jointangle-dependent moment arms based on the bone attachment points. The maximum tension available from a muscle is roughly a function of the physiological cross-sectional area $(P C S A)$ and muscle stretch (and, in non-isometric settings, the rate of lengthening or shortening).

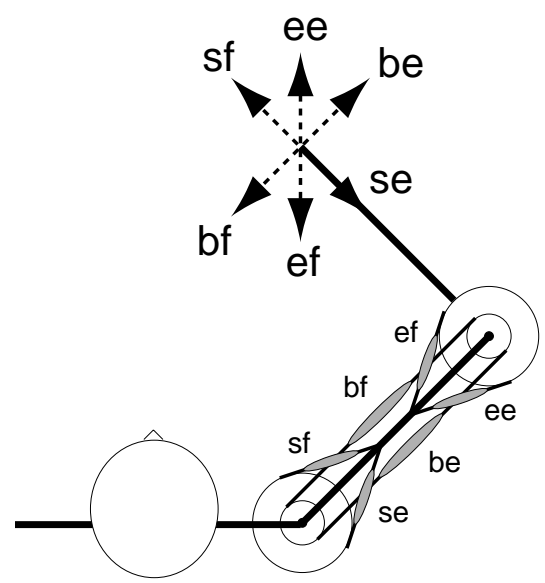

Figure 2: Hand force for each muscle group.

To simplify the model, we follow van Bolhuis and Gielen [14] and Gomi [4] and combine the muscles into six muscle groups: shoulder extensor and flexor, elbow extensor and flexor, and biarticular extensor and flexor. We define the muscle tension vector $\phi=$ $\left(\phi_{s e}, \phi_{s f}, \phi_{e e}, \phi_{e f}, \phi_{b e}, \phi_{b f}\right)^{T} \in \Re^{6}$ to capture the tension of each of these groups of muscles. All elements of the vector must be nonnegative, indicating that each muscle group is only capable of pulling. This simplification into muscle groups makes the assumption that all muscles in each group are activated proportionally [14]. With this model, the joint torques $\tau$ are obtained from the muscle tensions $\phi$ by

$$
\tau=A(\theta) \phi,
$$

where $A(\theta) \in \Re^{2 \times 6}$ is a matrix of joint-angle-dependent moment arms.

Figure 2 shows a model of the arm with these six muscle groups (adapted from [4]). By Equation (1), torque $\tau_{1}$ due to shoulder monoarticular muscles cause hand forces along the line of the forearm, torque $\tau_{2}$ due to elbow monoarticular muscles cause hand forces along the line through the shoulder, and biarticular muscles with $\tau_{1}=\tau_{2}$ generate hand forces parallel to the upper arm.

In our experiments, the subject was asked to hold stationary a handle that is free to slide on a smooth, linear rail, while the handle is pulled with a constant force along the rail. The subject must apply an opposing force to keep the handle stationary. While satisfying this one constraint, the subject has the following two kinds of freedoms:

(i) Constraint force. The subject may apply any desired force against the constraint without affecting the task. If the configuration space is $n$-dimensional, this implies $n-1$ constraint force freedoms. In our system, $n=2$, and there is one force freedom. 
(ii) Muscle load sharing. For a given total hand force $\mathbf{f}$ (constraint force plus the resisting force), there is an infinite set of muscle tension vectors $\phi$ that will generate it.

Gomi [4] and van Bolhuis and Gielen [14] have studied the muscle load sharing problem for isometric force generation in the planar arm system. Subjects were asked to apply specified forces at a fixed handle. EMG data of muscle activations were collected for each of the six muscle groups to determine how the load was distributed among the muscles. The results were then compared with the predictions of several different models for load sharing. These models are all based on minimization of some notion of "effort."

Our experiments differ in that the subject is only required to apply a specified force in a single direction (along the direction of motion freedom of the constraint). The subject is free to choose any force in the orthogonal subspace, to simplify the task. Our goal is to model how subjects choose forces in this orthogonal space. We simply measure the applied force against the constraint; we do not take EMG data.

We will consider the following models for interaction with the constraint. Each of these models is based on the minimization of some quantity. Some of these models were considered for isometric force generation in [4, 14]. The first two models ignore the muscle load-sharing issue.

- HAND Hand force magnitude $\|\mathbf{f}\|$ is minimized. According to this model, the subject applies only resisting forces. The constraint force is zero.

- T2 Torque squared, $\sum_{i} \tau_{i}^{2}$. For a robot arm with identical motors at the shoulder and elbow, this solution minimizes the electrical power to the motors. A geometric interpretation of this model is given in Figure 3.

- MT1 Muscle tension, $\sum_{i} \phi_{i}, i \in\{s e, s f, e e, e f$, $b e, b f\}$. This model for force generation was proposed by Yeo [17].

- MT2 Muscle tension squared, $\sum_{i} \phi_{i}^{2}$. Nelson [10] and Hogan [6] suggest that metabolic power consumed by a muscle is proportional to the square of muscle force.

- MT3 Muscle tension cubed, $\sum_{i} \phi_{i}^{3}$.

- MS1 Muscle stress, $\sum_{i} \phi_{i} / P C S A_{i}$, where $P C S A_{i}$ is the physiological cross-sectional area of muscle $i$. This is a measure of the activation of the muscle.

- MS2 Muscle stress squared, $\sum_{i}\left(\phi_{i} / P C S A_{i}\right)^{2}$.

- MS3 Muscle stress cubed, $\sum_{i}\left(\phi_{i} / P C S A_{i}\right)^{3}$. There is some evidence that muscle endurance time is inversely proportional to $(\phi / P C S A)^{3}$ [12].

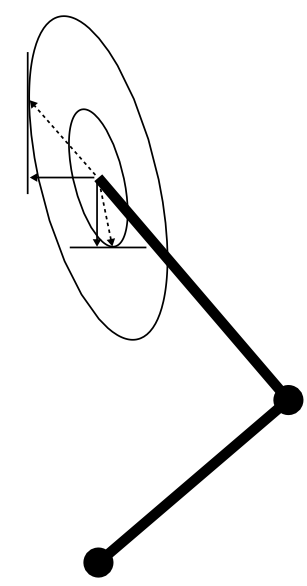

Figure 3: The iso-cost torque circles of model T2 in the $\left(\tau_{1}, \tau_{2}\right)$ space become iso-cost force ellipses in the hand force space $\left(f_{x}, f_{y}\right)$, as a function of the joint angles. The solid arrows in the figure represent example hand forces along the rail necessary to resist motion of the handle. The dashed arrows indicate the optimal hand forces which provide the resisting forces. The normal forces are chosen to place the force vectors on the smallest possible ellipses.

The linear models MT1 and MS1 tend to predict activation of only one of the muscle groups for a given task, while higher-order models predict greater sharing of the load across the muscle groups. Any model other than HAND indicates that the subject chooses a strategy that would not be possible without the guide.

The experimental results of the next section are compared to the predictions of each of these models. To obtain the prediction for model T2, let $\alpha \hat{\mathbf{f}}_{n}$ represent the force applied against the constraint, where $\hat{\mathbf{f}}_{n}$ is a unit vector normal to the tangential force $\mathbf{f}_{t}$ applied by the subject to resist motion. Then $\alpha$ is obtained by solving

$$
\frac{d}{d \alpha}\left(J(\theta)^{T}\left(\mathbf{f}_{t}+\alpha \hat{\mathbf{f}}_{n}\right)\right)^{2}=0 .
$$

To obtain the predictions of models MT $k$ and MS $k, k=$ $1,2,3$, we solve for the tension vector $\phi$ minimizing the objective function, subject to $\phi \geq 0$ (all muscles pulling) and

$$
\left(\left(J(\theta)^{T}\right)^{-1} A(\theta) \phi\right)^{T}\left(\mathbf{f}_{t} /\left\|\mathbf{f}_{t}\right\|\right)=\left\|\mathbf{f}_{t}\right\|,
$$

requiring that the tangential force be equal to $\mathbf{f}_{t}$. This problem is a linear programming problem for $k=1$ and a nonlinear optimization for $k=2,3$. We solved all of these optimizations using CFSQP [7], C code implementing sequential quadratic programming.

Following Gomi [4], Table 1 gives the physiological cross-sectional area $P C S A$ used for each of the muscles. It also gives the elements of the matrix $A$ of moment arms. 


\begin{tabular}{|r|rrrrrr|}
\hline & $s e$ & $s f$ & $e e$ & $e f$ & $b e$ & $b f$ \\
\hline$P C S A\left(\mathrm{~cm}^{2}\right)$ & 38.71 & 19.36 & 7.75 & 10.3 & 3.87 & 3.23 \\
\hline$A_{1 i}(\mathrm{~cm})$ & -3.52 & 4.37 & 0 & 0 & -2.54 & 2.9 \\
$A_{2 i}(\mathrm{~cm})$ & 0.0 & 0.0 & -2.03 & 2.75 & -3.05 & 4.32 \\
\hline
\end{tabular}

Table 1: Physiological parameters of the muscle groups used in the arm model.

\section{Experiments}

\subsection{Setup}

Subjects were seated in a custom-made high-backed chair with an adjustable seat, to raise or lower the height of the shoulder plane based on the height of the subject. To fix the shoulder location, subjects were restrained by a fourpoint harness. The wrist was immobilized by an over-thecounter wrist cuff, and the subject grasped a vertical handle on a slider on a horizontal low-friction linear track (Thomson twin shaft 2BA linear ball guide \#13). The handle can spin freely about a vertical axis, and a support plate is attached to the handle to support the forearm. This support maintains the arm in a horizontal plane throughout the experiments without fatiguing the shoulder.

A six-axis force sensor (ATI-AI Gamma 15-50) sits between the handle and the slider and was used to measure forces against the rail. Cables attached to the slider pass through a series of pulleys, allowing weights to be suspended at either end of the rail to create a pulling force along the rail (Figure 4). The rail is used as a linear potentiometer, with a wiper at the slider to measure the position along the rail. The actual and desired positions of the slider were shown to the subject on a computer monitor. Force information was not displayed.

\subsection{Protocol}

The subject was asked to hold the handle stationary, at a location indicated on the monitor, as different weights were hung from the cables. The weights included one light weight pulling to the left on the rail, one heavy weight pulling to the left, one light weight pulling to the right, and one heavy weight pulling to the right. After hanging a weight and after the subject stabilized the position of the handle, forces normal to the rail were recorded for two seconds. The sequence of four weights was repeated 10 times for each of six configurations of the chair relative to the rail. These six configurations allowed us to test three different positions of the handle relative to the shoulder, and two different orientations of the rail at each position. The three positions were a distance $45 \mathrm{~cm}$ from the shoulder, at 60, 90, and 120 degrees from the line passing through both shoulders (see Figure 5). The rail was oriented either along the line through the shoulder (denoted "parallel"), or along a line perpendicular to the line through the shoulder (denoted "perpendicular").
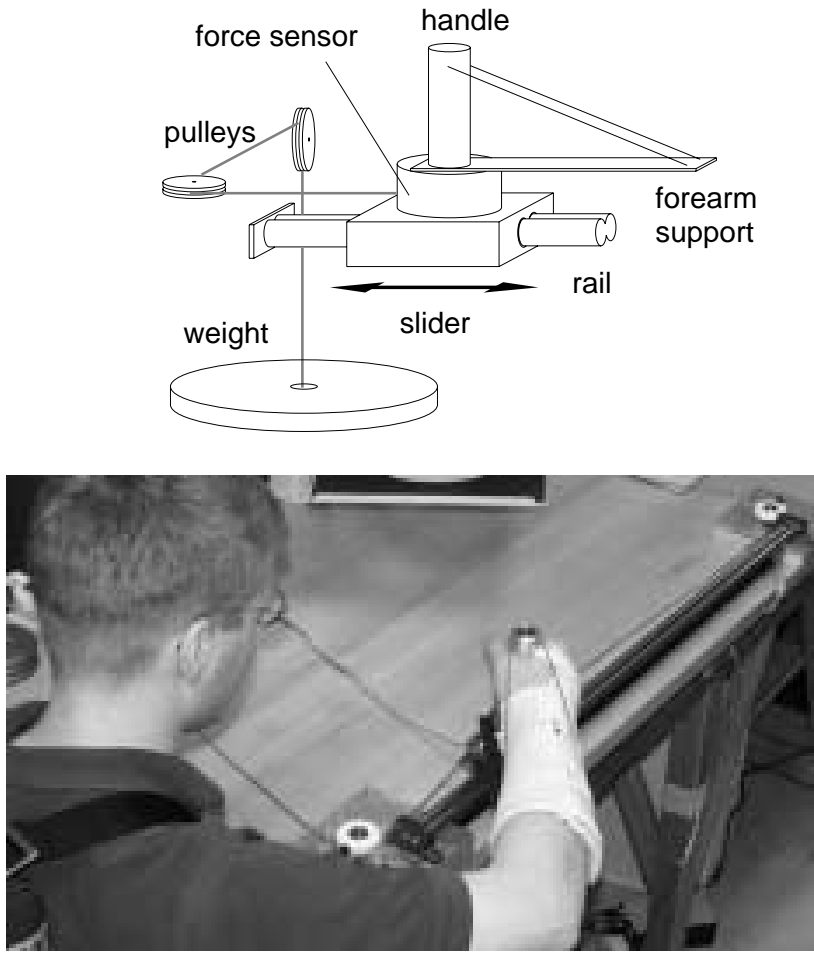

Figure 4: A drawing of the slider and rail setup, and a subject during an experiment.

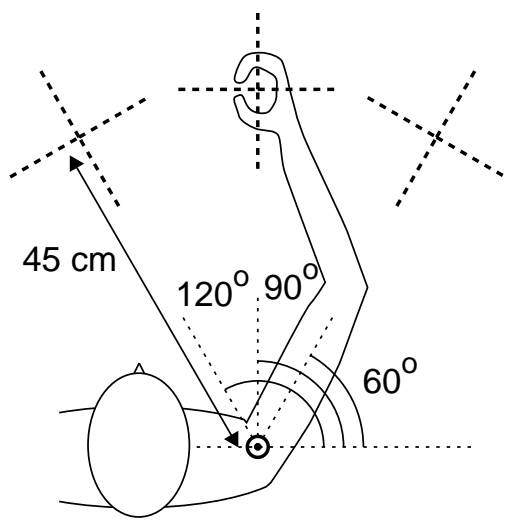

Figure 5: The three positions of the slider during the experiments. The rail is oriented either through the shoulder joint (parallel) or perpendicular.

The heavy and light weights varied for each subject, depending on the maximum load they were comfortable suspending during pre-recording trials. The heavy weight was approximately $80 \%$ of the heaviest weight the subject felt comfortable suspending at 120 degrees and perpendicular, and the light weight was approximately $50 \%$ of the heavy weight. 


\begin{tabular}{|r|r|r|r|r|r|r|}
\hline & Sex & Age & $L_{1}(\mathrm{~cm})$ & $L_{2}(\mathrm{~cm})$ & Heavy $(\mathrm{kg})$ & Light $(\mathrm{kg})$ \\
\hline 1 & $\mathrm{M}$ & 24 & 30.2 & 38.6 & 4.3 & 2.2 \\
\hline 2 & $\mathrm{~F}$ & 26 & 32.0 & 31.5 & 2.8 & 1.4 \\
\hline 3 & $\mathrm{M}$ & 25 & 26.9 & 31.6 & 3.25 & 1.75 \\
\hline 4 & $\mathrm{M}$ & 18 & 30.2 & 33.3 & 3.6 & 1.8 \\
\hline 5 & $\mathrm{~F}$ & 22 & 28.0 & 32.3 & 2.8 & 1.4 \\
\hline
\end{tabular}

Table 2: The upper and forearm lengths of the five subjects and the weights used in the experiments.

Subjects were told to suspend the weight as naturally and comfortably as possible, and not to cocontract to stiffen the position of the handle. Subjects were permitted to take a break at any time to prevent fatigue. Fatigue was minimized by the short durations of each experiment and by cycling through lighter and heavier weights. The total time for each subject was about 90 minutes. The protocol was approved by the Northwestern University Institutional Review Board.

\subsection{Results}

We recruited five healthy right-handed subjects for our experiments (Table 2). Figure 6 shows the forces at the hand predicted by seven of the eight models for subject 1 . For each model there are six plots, corresponding to the three different rail positions with two different rail orientations, as in Figure 5. For each plot, there are four force vectors shown, corresponding to the four different weights pulling along the rail (two in one direction, two in the other). Each force vector is the sum of the force to resist the pulling force along the rail, plus any force normal to the rail. Not shown is the HAND model, which predicts zero normal force (all forces are along the dotted line of the rail).

For each model, the direction (angle) of the predicted force is independent of the magnitude of the pulling force, but dependent on the direction of the pulling force. T2 predicts significantly larger normal forces for the perpendicular orientations than the parallel orientations. The linear model MT1 predicts larger normal forces than MT2 and MT3 because a single muscle group tends to be utilized for the linear models, whereas the redundant actuation is used in MT2 and MT3 to decrease the magnitude of the normal forces. The nonlinearity in the MT $k$ models, not evident in the T2 model, comes from the asymmetry in the extensors and flexors. In the MSk models, the large PCSA value for the shoulder extensor and flexor predicts much larger forces at these muscles than any other. This preference for the shoulder actuators results in large normal forces in both the parallel and perpendicular orientations. Predicted differences between squared and cubed models were generally small.

The experimental results for the five subjects are shown in Figure 7. Each force vector shows the average hand force over the ten trials. At the head of each vector is a set of error bars indicating the standard deviation of the normal

\begin{tabular}{|c|r|r|r|r|r|}
\hline model & Subj1 & Subj2 & Subj3 & Subj4 & Subj5 \\
\hline HAND & 18.5 & 14.3 & 17.8 & 17.2 & 13.2 \\
\hline T2 & 13.9 & 16.6 & 28.1 & 20.6 & 23.4 \\
\hline MT1 & 20.3 & 30.0 & 31.6 & 23.9 & 27.1 \\
\hline MT2 & 16.5 & 11.4 & 19.2 & 13.6 & 7.9 \\
\hline MT3 & 14.8 & 13.2 & 20.0 & 15.1 & 7.9 \\
\hline MS1 & 29.9 & 32.4 & 40.6 & 34.4 & 34.4 \\
\hline MS2 & 28.2 & 29.4 & 38.5 & 32.0 & 32.2 \\
\hline MS3 & 26.3 & 26.9 & 36.4 & 29.8 & 30.0 \\
\hline
\end{tabular}

Table 3: The average error between the angle of the total force (tangential plus normal force) predicted by the models and the actual angle of the force applied by the subject. The average error is expressed in degrees.

force over the ten trials. As in the models, it appears that the angle of the total hand force is essentially independent of the pulling force magnitude. The apparent nonlinearity in the force angle may be a result of asymmetry in the arm structure.

One clear observation we can make is that subjects often choose to apply forces against the constraint, even though these forces are unnecessary for the task. Table 3 gives a measure of how well each of the models predicted the experimental results. For a given model and subject, the table entry is $\frac{1}{n} \sum_{i=1}^{n}\left|\beta_{i}^{p}-\beta_{i}\right|$, where $\beta_{i}^{p}$ is the angle of the total force (tangential plus normal force) predicted by the model for trial $i, \beta_{i}$ is the actual angle of the force applied by the subject in trial $i$, and $i$ ranges over all $n$ trials (all experiments for each of the three rail positions and two rail orientations). For each subject, the MT2 and MT3 models provided a better fit than the MT1 model, perhaps indicating that the load sharing in the MT2 and MT3 models is an important aspect of natural force generation. For four of the five subjects, the HAND, MT2, and MT3 models provide a better fit to the data than the T2 model, perhaps indicating that the actuator redundancy in these models (not present in the robotic T2 model) is important in predicting the interaction forces. The MS $k$ models were poor predictors of the interaction forces, though some evidence for the MS2 model was found in both [4, 14] for isometric force generation. We note, however, that the PCSA values for the shoulder muscle groups used in [4] (which we adopted) were much larger than those used in [14]. Smaller values for the shoulder muscle groups would result in smaller shoulder forces and smaller predicted normal forces.

The experimental results are insufficient to definitively select one model as preferable to all others. We are currently following up these experiments with more detailed experiments studying constraint forces with the rail angles sampled at a finer resolution. These experiments are described in Section 5. 


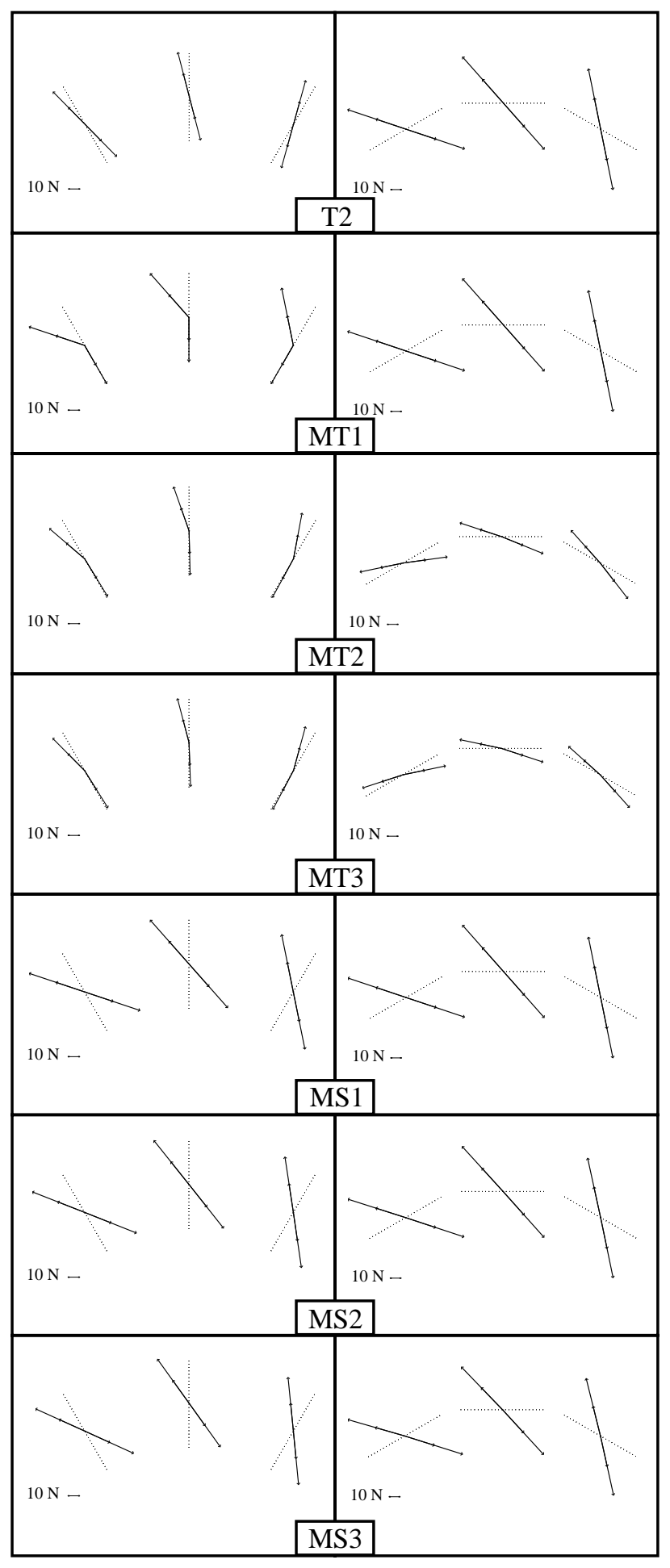

Figure 6: Predicted applied force vectors (resisting plus normal force) for subject 1 for different models. The left column is for the rail oriented through the shoulder (parallel), and the right column is for perpendicular orientations. The predictions for other subjects may look different due to different upper and forearm lengths.

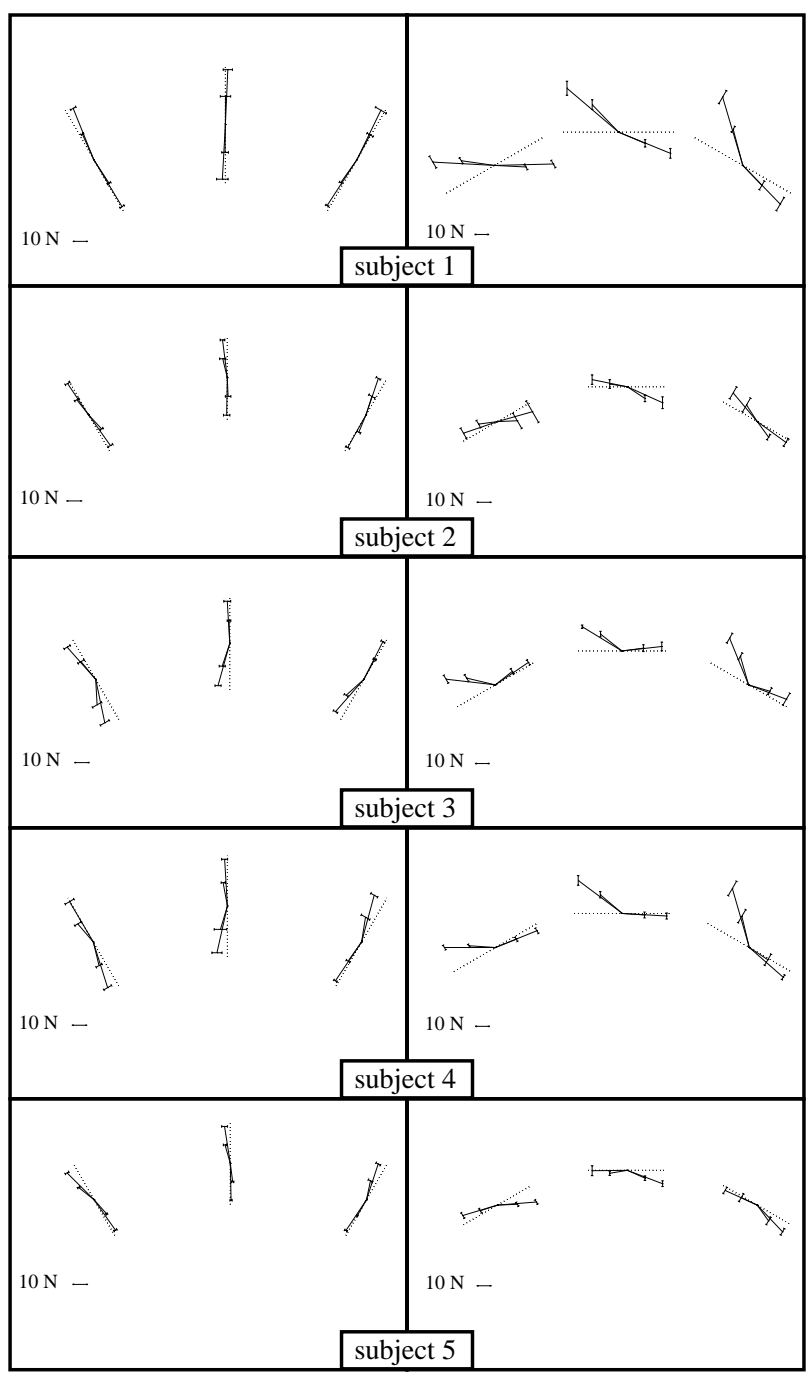

Figure 7: Experimental results for the five subjects. Each individual plot shows the average subject force vector for the four different pulling forces along the rail. The error bars at the end of each vector show the standard deviation of the normal force over the ten trials with each weight.

Overall, it appears that subjects apply more force against the rail for the perpendicular orientations of the rail than the parallel orientations. The required joint torques for the perpendicular orientations are also larger (see, e.g., Figure 3).

\section{Discussion}

Previous work in constrained arm motion includes Gomi's study of hand stiffness ellipses during point-to-point arm motion [3]. Subjects were constrained to move on a line by a planar manipulandum robot enforcing a high servo gain normal to the virtual rail. Perturbations were used to estimate the stiffness at the hand during the motion. Gomi found 
that stiffness in the direction normal to the constraint was greatly reduced compared to the stiffness in that direction during unconstrained motions. One possible explanation is that subjects adapted to the virtual constraint by changing joint stiffnesses to reduce interaction forces (comply) with the environment. Our experiments show that in certain situations, subjects choose to maintain interaction forces with the environment even though they have no effect on the task.

The load sharing models studied in this paper are based on $P C S A$ and moment arm data taken from [4]. The PCSA values for the shoulder muscle groups used in [14] are dramatically smaller, which predicts less shoulder activation in the MS $k$ models, and as a result less constraint force. The PCSA and moment arm data cannot be reliably measured for each subject individually, and in any case these are approximations for the complex set of individual muscles. Beer et al. [2] have empirically studied maximal torque envelopes of elbow and shoulder flexion and extension at different arm positions. Maximum torque envelopes are anisotropic and not simply circles centered at the origin of the $\left(\tau_{1}, \tau_{2}\right)$ space. Such data could be used as the basis for a different model of natural arm interaction with a rail.

Other models for interaction with a constraint could be considered. For instance, it may be difficult to precisely coordinate force direction, but less difficult to control force magnitude. Kinematic constraints offer the user the opportunity to ramp up force magnitude, with little concern for force direction, until the slider is stabilized. In this sense, it would be interesting to study the time evolution of the hand force as the slider is stabilized.

Although normal forces across ten trials are similar for a single subject, there is some variation, as visible in the standard deviation bars in Figure 7. This may be due to natural motor output variation, but further investigation is necessary. Convergence of the normal forces, or "learning" to use the constraint, did not appear to occur during the trials.

Subjects were asked to minimize cocontraction of muscles, and none of the models predict cocontraction. Significant cocontraction is wasteful metabolically but simplifies the posture maintenance problem.

Friction in the system came from the bearings in the pulleys and from the slider-rail contact. We estimate friction in the pulleys provide about $1.5 \mathrm{~N}$ of resistance to motion along the rail, and the friction coefficient between the slider and rail is about $\mu=0.05$. This implies that up to $5 \%$ of the normal force is available as tangential force to resist slider motion. These frictional forces were considered insignificant in the analysis.

\section{Conclusion and Future Work}

Each of the eight models considered in this paper defines iso-cost force contours in the hand frame for a particular arm configuration. For the T2 model, the iso-cost contours are
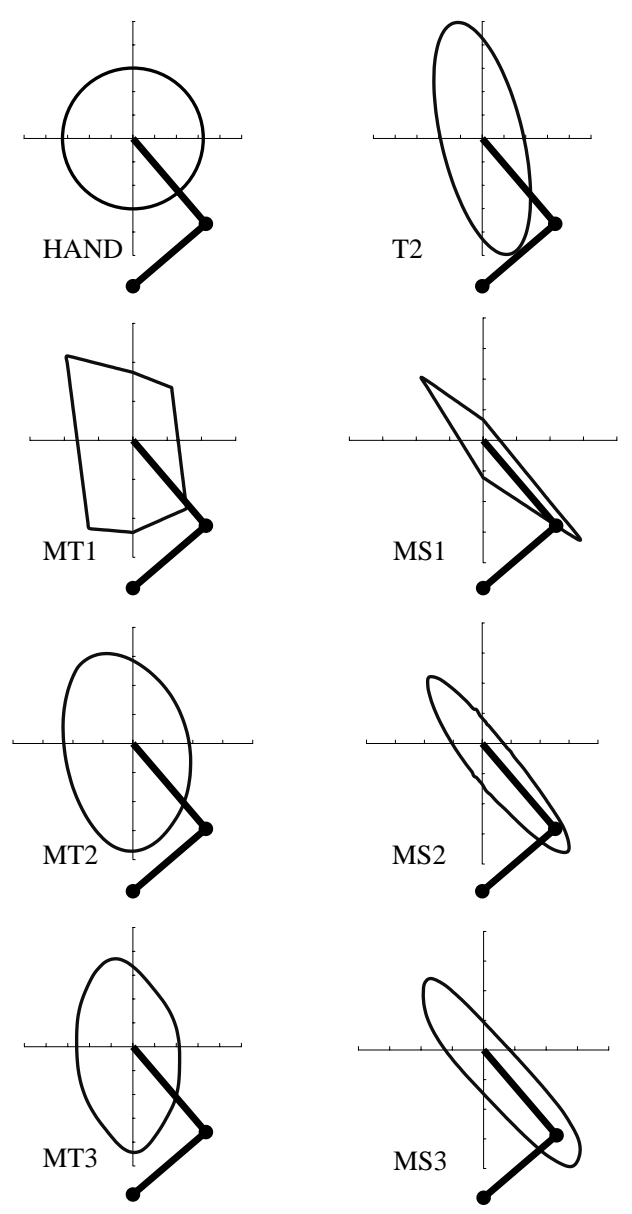

Figure 8: The iso-cost force contours for the eight different models for a hand position at $(0,45 \mathrm{~cm})$ in the shoulder frame, $L_{1}=30 \mathrm{~cm}, L_{2}=35 \mathrm{~cm}$, and the physiological parameters in Table 1. For a particular model, a point on the iso-cost contour corresponds to a force vector with its origin at the hand. The shapes of the contours are independent of the cost; the contours simply scale as the cost is varied.

ellipses which can be found in closed form (Figure 3 ). The iso-cost contours for the HAND model are simply circles centered at the origin. The iso-cost contours for the other models can be found numerically (Figure 8). The shapes of the contours are independent of the cost; the contours simply scale as the cost is varied. The linear models MT1 and MS1 result in polygonal iso-cost contours; the other models have strictly convex iso-cost contours. Note the strong anisotropy of the MSk iso-cost contours, due to the large PCSA of the uniarticular shoulder muscles.

As the direction of the tangential force $\mathbf{f}_{t}$ applied by the human along the rail varies between $0^{\circ}$ and $360^{\circ}$, holding $\left|\mathbf{f}_{t}\right|$ constant, the different models predict different normal forces. Figure 9 shows predicted normal forces for the mod- 


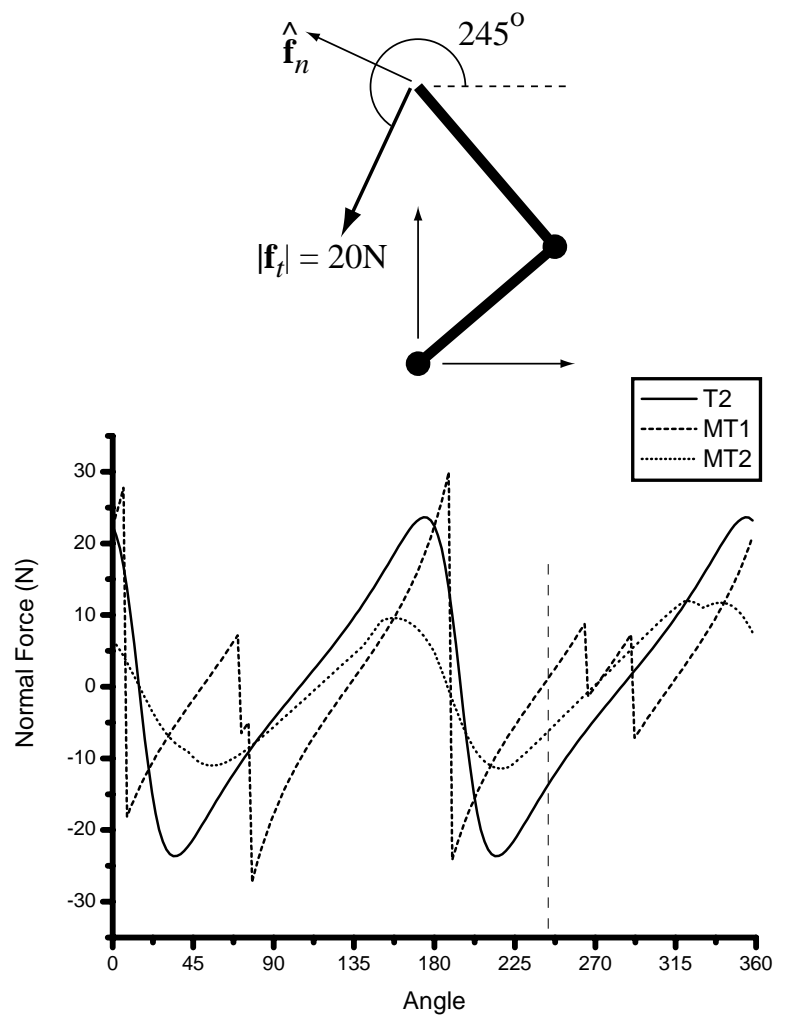

Figure 9: The normal force predicted by three different models, T2, MT1, and MT2. The magnitude of the tangential force $\mathbf{f}_{t}$ (along the rail) applied by the human is $20 \mathrm{~N}$, and the direction of $\mathbf{f}_{t}$ is varied between $0^{\circ}$ and $360^{\circ}$. The positive direction of the normal force is defined as $90^{\circ}$ clockwise of the direction of the tangential force, indicated by the vector $\hat{\mathbf{f}}_{n}$ in the figure. The arm is identical to, and at the same configuration as, the arm in Figure 8. The figure shows a tangential force at $245^{\circ}$, and the three models predict different normal forces.

els T2, MT1, and MT2 for the arm of Figure 8. Discontinuities in the normal force plot for the model MT1 arise from "flats" (sections of zero curvature) in the iso-cost contours (Figure 8). Zero-crossings in the normal force plots occur at radial local maxima and minima of the iso-cost contours. The HAND model predicts zero normal force for all tangential force directions.

Our current work is to measure normal forces at a large number of constraint angles to generate experimental normal force plots similar to those in Figure 9. Such plots will allow us to identify features of the iso-cost contours, better differentiate the predictive ability of the different models, and develop better models for static interaction with a constraint. Our future work will study dynamic interactions with kinematic constraints. Models of natural interaction with a constraint will be used to design robotic virtual guides for human-robot collaborative manipulation.

\section{Acknowledgments}

We would like to thank Sandro Mussa-Ivaldi, Jules Dewald, and Joe Hidler for stimulating discussions on this topic, as well as the entire robotics lab at the Northwestern Rehabilitation Institute in Chicago, especially Jon Dingwell, Chris Mah, and Jim Patton. Thanks to Vik Chib for data analysis and Peng Pan for Figures 8 and 9. This work was funded by NSF grants IIS-9875469 and IIS-0082957.

\section{References}

[1] K. N. An, F. C. Hui, B. F. Morrey, R. L. Linscheid, and E. Y. Chao. Muscles across the elbow joint: a biomechanical analysis. Journal of Biomechanics, 14(10):659-669, 1981.

[2] R. F. Beer, J. D. Given, and J. P. A. Dewald. Task-dependent weakness at the elbow in patients with hemiparesis. Archives of Physical Medicine and Rehabilitation, 80(7):766-772, 1999.

[3] H. Gomi. Anisotropic stiffness reduction during constrained multijoint arm movement. In Proceedings of the 20th Annual International Conference of the IEEE Engineering in Medicine and Biology Society, volume 20, pages 2336-2337, 1998.

[4] H. Gomi. Minimum principles for muscle-coordination evaluated by the directional preference of muscle activation during multijoint-arm force regulation tasks. Technical report, NTT Communication Science Labs, 2000.

[5] F. C. T. van der Helm. A finite element musculoskeletal model of the shoulder mechanism. Journal of Biomechanics, 25(7):551-569, 1994

[6] N. Hogan. Adaptive control of mechanical impedance by coactivation of antagonist muscles. IEEE Transactions on Automatic Control, 29(8):681-690, August 1984

[7] C. Lawrence, J. L. Zhou, and A. L. Tits. User's guide for CFSQP version 2.3. Institute for Systems Research 94-16, University of Maryland, 1994.

[8] S. G. Meek, J. E. Wood, and S. C. Jacobsen. Model-based, multimuscle emg control of upper-extremity prostheses. In J. M. Winters and S. L-Y. Woo, editors, Multiple Muscle Systems: Biomechanics and Movement Organization. Springer-Verlag, 1990.

[9] W. M. Murray, T. S. Buchanan, and S. L. Delp. The isometric functional capacity of muscles that cross the elbow. Journal of Biomechanics, 33:943-952, 2000.

[10] W. L. Nelson. Physical principles for economies of skilled movements. Biological Cybernetics, 46:135-147, 1983.

[11] P. Pigeon, L. Yahia, and A. G. Feldman. Moment arms and lengths of human upper limb muscles as functions of joint angles. Journal of Biomechanics, 29(10):1365-1370, 1996.

[12] B. I. Prilutsky, T. Isaka, A. M. Albrecht, and R. J. Gregor. Is coordination of two-joint leg muscles during load lifting consistent with the strategy of minimum fatigue? Journal of Biomechanics, 31:10251034, 1998.

[13] M. M. Svinin, K. Ohta, Z. W. Luo, and S. Hosoe. Understanding of human movements in crank rotation. In IEEE/RSJ International Conference on Intelligent Robots and Systems, pages 2105-2110, 2001.

[14] B. M. van Bolhuis and C.C.A.M. Gielen. A comparison of models explaining muscle activation patterns for isometric contractions. Biological Cybernetics, 81:249-261, 1999.

[15] W. Wannasuphoprasit, R. B. Gillespie, J. E. Colgate, and M. A Peshkin. Cobot control. In IEEE International Conference on Robotics and Automation, pages 3571-3577, 1997

[16] J. E. Wood, S. G. Meek, and S. C. Jacobsen. Quantitation of human shoulder anatomy for prosthetic arm control - I. Surface modeling. Journal of Biomechanics, 22(3):273-292, 1989.

[17] B. P. Yeo. Investigations concerning the principle of minimal total muscular force. Journal of Biomechanics, 9:413-416, 1976. 